\title{
The Quantitative Measurement of Forces Acting on a Powder Bed During a Specific Mechanical Process for Preparation of Composite Particles ${ }^{\dagger}$
}

\author{
Hiroyuki Yoshino, Yoshihiro Taya \\ and Hisashi Kaga \\ Hokkaido Industrial Technology Center* \\ Ken-ichi Mukaida \\ Muroran Inst. of Technol**
}

\begin{abstract}
Using the mechanical processing method called mechanofusion to prepare composite particles, the authors conducted a study on the preparation of metal-ceramic composite particles in a vacuum. The major principle in forming composite particles is the application of mechanical forces to a mixed powder in the apparatus used. The measurement of those forces, however, has seldom been attempted. Here we used strain gauges in an attempt to quantitatively measure the forces acting on a powder bed.

An experiment using glass beads as the raw material showed that (1) if the drum's rotational speed is high and the powder charge is large, great compressive and frictional forces are generated, and (2) compressive force is several times larger than frictional force. These forces were measured during the preparation of composite particles using $\mathrm{Cu}-\mathrm{Al}_{2} \mathrm{O}_{3}$ mixed powder under reduced pressures, and both forces appeared to be higher when atmospheric pressure was reduced.
\end{abstract}

\section{Introduction}

There are now many attempts to create composite particles by affixing one kind of particle to the surface of a different kind, and these attempts often use devices derived from pulverizers and mixers. Methods using such mills to make composite particles are termed mechanical compositing processes. One of these methods, called mechanofusion, applies to powders such forces as compression, friction, shearing, and rotation between a rotating drum (mortar) and a fixed inner piece (pestle), and in this way composite particles are said to be formed ${ }^{1,2}$. Researchers are making the most of the simplic ity, low contamination, and other advantages of this process to pursue research and development, and there are hopes for its industrialization ${ }^{3}$.

The authors and others have demonstrated that, in the formation of composite particles by mechani-

\footnotetext{
* 379 Kikyo-cho, Hakodate 041

**27-1 Mizumoto, Muroran 050

'This report was originally printed in J. Soc. Powder Technology, Japan. 32, 866(1995) in Japanese, before being translated into English by KONA Editiorial Committee with the permission of the editiorial committee of the Soc. Powder Technology, Japan.
}

cal processing under a vacuum using $\mathrm{Cu}-\mathrm{Al}_{2} \mathrm{O}_{3}$ mixed powder, the lower the residual pressure, the greater the extent to which compositing proceeds ${ }^{4.5}$. Analyses of particle surfaces revealed that the reduced pressure could alleviate the process of oxidation $^{6,7}$.

Even though forces acting on powders are a main factor in compositing, there is very little research on analyses of these forces in compositing processes $^{8}$, and even research reports on composit. ing that have appeared thus far include only a few that, in terms of test conditions in compositing, explicitly present the factors influencing these forces, instead writing only about the drum's rotational speed. From now on, however, it will be necessary to perform quantitative evaluations of these forces in order not only to scale up the devices used in the manufacturing process of composite particles by mechanical processing, but also to elucidate in detail the process by which compositing occurs.

This research involved developing a way to measure the frictional force and compressive force generated on the tip of the inner piece used in this mechanical process, and measuring the forces using 
glass 1 beads, as well as assessing the soundness of the measurement method with the coincidence of calculated and measured values for power consumption. The research also examines, in accordance with the results of force measurements, the effect of a vacuum on compositing using the $\mathrm{Cu}-\mathrm{Al}_{2} \mathrm{O}_{3}$ mixed powder under a vacuum.

\section{Experimental}

\subsection{Measurement Method Principle}

The device used in the experiment was a model AM-15AF mechanofusion apparatus (made by Hosokawa Micron Corporation), to which we had added functions to perform composite processing under a high vacuum. Fig. 1 shows detailed diagrams. The apparatus is designed to load $150 \mathrm{cc}$ of powder and to directly exhaust the container interior during mechanical processing. It can maintain a high vacuum on the order of $10^{-2} \mathrm{~Pa}$.

We measured the frictional force and compressive force generated on the part of the inner piece that makes contact with the powder while processing it, and determined the central point of contact with the powder on the inner piece head where these forces begin to arise (the mechanical action origin).

Figs. 2(a) and (b) schematically show strain gauge positions, while Fig. 2(c) schematically shows the relation among forces at point $\mathrm{PL}$, the center of contact with the powder.

The equations below are used to calculate the forces generated where the inner piece and the powder make contact, with calculation based on the stress that is determined through strain measured by the strain gauges on the inner piece's arm.

$$
\begin{aligned}
& F_{c}=f_{c} \cos \theta-f_{f} \sin \theta \\
& F_{f}=f_{f} \cos \theta+f_{c} \sin \theta
\end{aligned}
$$

Where:

$f_{c}$ is compressive force acting on the area of contact,

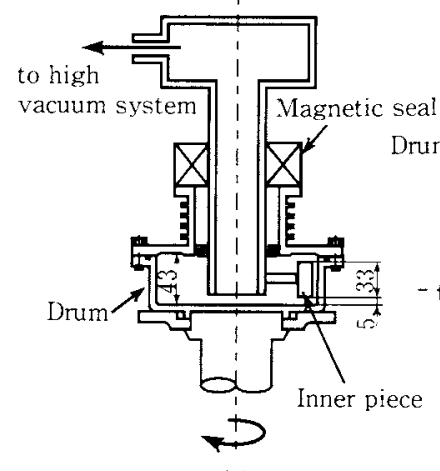

(a)
Drum rotation

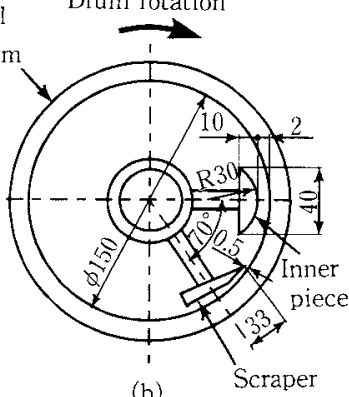

(b)
Fig. 1 Schematic diagrams of vacuum mechanical preparation apparatus (a) and of the drum section (b). (mm) $f_{f}$ is frictional force acting on the area of contact, $F_{c}$ is the horizontal component of the inner piece's arm, and

$F_{f}$ is the vertical component of the arm.

Stresses in these locations, i.e., $\sigma_{n}, \sigma_{n}{ }^{\prime}(\mathrm{n}=1,2,3)$, and $\sigma_{4}, \sigma_{4}^{\prime}$, are as follows.

$$
\begin{aligned}
& \sigma_{n}=-\frac{F_{c}}{A}-\frac{F_{c} l}{Z}+\frac{F_{f} l_{n}}{Z} \\
& \sigma_{n}{ }^{\prime}=-\frac{F_{c}}{A}+\frac{F_{c} l}{Z}-\frac{F_{f} l_{n}}{Z} \\
& \sigma_{4}=-\frac{F_{c}}{A}-\frac{F_{c} l^{\prime}}{Z} \\
& \sigma_{4}{ }^{\prime}=-\frac{F_{c}}{A}+\frac{F_{c} l^{\prime}}{Z}
\end{aligned}
$$

Solving Equations (3) through (6) for $F_{c}$ and $F_{f}$ yields :

$$
\begin{gathered}
F_{c}=-A \frac{\sigma_{4}+\sigma_{4}{ }^{\prime}}{2} \\
F_{f}=\frac{Z}{\left(l_{3}-l_{1}\right)} \frac{\left(\sigma_{3}-\sigma_{3}{ }^{\prime}\right)-\left(\sigma_{1}-\sigma_{1}{ }^{\prime}\right)}{4}
\end{gathered}
$$

And because

$$
\sin \theta=\frac{l}{R}, \cos \theta=\frac{\sqrt{R^{2}-l^{2}}}{R}
$$

we obtain from Equations (1) through (9)

$$
f_{c}=F_{c} \frac{\sqrt{R^{2}-l^{2}}}{R}+F_{f} \frac{l}{R}
$$

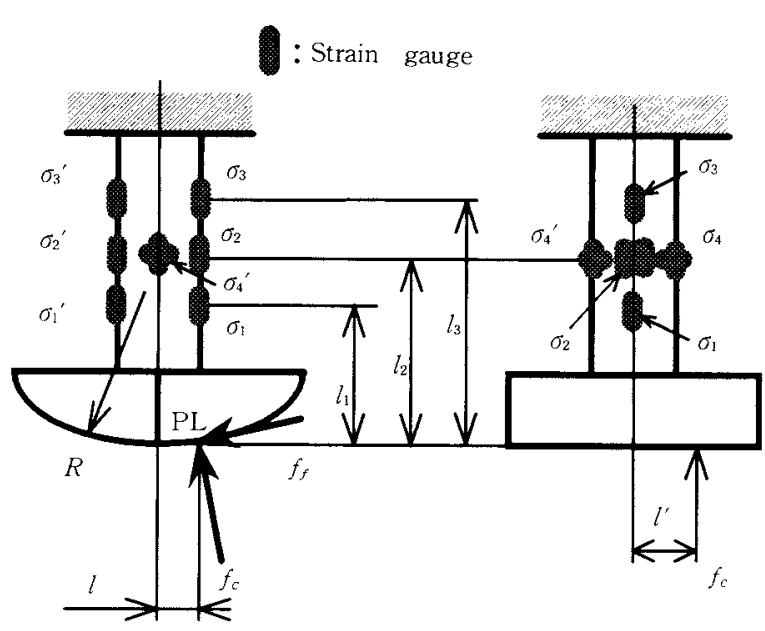

(a)

(b)

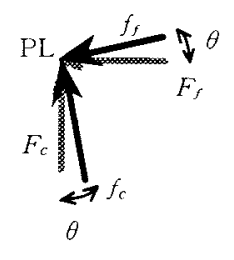

(c)

Fig. 2 The position of the strain gauges in Fig. (a) and (b), and the relation of forces at the point PL in Fig. (c). 


$$
\begin{aligned}
& f_{f}=F_{f} \frac{\sqrt{R^{2}-l^{2}}}{R}-F_{c} \frac{l}{R} \\
& l=\frac{Z}{F_{c}}\left(\frac{F_{f} l_{2}}{Z}-\frac{\sigma_{2}-\sigma_{2}{ }^{\prime}}{2}\right)
\end{aligned}
$$

Thus, measuring $\sigma_{1}$ through $\sigma_{4}$ and $\sigma_{1}{ }^{\prime}$ through $\sigma_{4}{ }^{\prime}$ makes it possible to find compressive force $F_{c}$, frictional force $F_{f}$, and the distance from the inner piece center to the center of contact. This measurement method involves using the four active gauge method $^{9}$ with temperature-compensating circuits, forming three bridges, measuring $\left(\sigma_{3}-\sigma_{3}{ }^{\prime}\right)-\left(\sigma_{1}-\right.$ $\left.\sigma_{1}{ }^{\prime}\right),\left(\sigma_{2}-\sigma_{2}{ }^{\prime}\right)$, and $\left(\sigma_{4}+\sigma_{4}{ }^{\prime}\right)$, and using Equations (7) through (12) to determine the frictional force and compressive force arising at the area of contact between the inner piece and the powder, and the distance $l$ to the mechanical action origin.

The strain gauges shown in Fig. 2 also register apparent strain owing to structural effects, i.e., frictional heat from the tip of the inner piece and from drum atmosphere. Although all bridges have temperature-compensating circuits as mentioned above, the microdisplacement structure makes it difficult to completely cancel out the effects of temperature. For that reason we attached thermocouples near the strain gauges to measure gauge temperature and compensate for the apparent displacement due to temperature rise.

\subsection{Basic Evaluation of Measurement Method}

We mounted the inner piece shown in Fig. 2 on a stationary bracket, imposed a load on the inner piece's tip, and measured the voltage output from each bridge at that time. Figs. 3 and 4 show the basic characteristics of the imposed load and voltage output.

In Fig. 3 we imposed a load on the inner piece head at a right angle (parallel to the arm) and measured the relationship of voltage output to the compressive load. As the compressive load increases, the voltage output of $\left(\sigma_{4}+\sigma_{4}{ }^{\prime}\right)$ increases linearly. By contrast, the voltage outputs of $\left(\sigma_{3}-\right.$ $\left.\sigma_{3}{ }^{\prime}\right)-\left(\sigma_{1}-\sigma_{1}{ }^{\prime}\right)$ and $\left(\sigma_{2}-\sigma_{2}{ }^{\prime}\right)$ are $0 \mathrm{~V}$, showing that the bending component has been canceled.

Fig. 4 shows the relationship of voltage output to bending load when a load is imposed on the inner piece head in a tangential direction (perpendicular to the inner piece arm). Voltage outputs of $\left(\sigma_{3}-\right.$ $\left.\sigma_{3}{ }^{\prime}\right)-\left(\sigma_{1}-\sigma_{1}{ }^{\prime}\right)$ and $\left(\sigma_{2}-\sigma_{2}{ }^{\prime}\right)$ increase linearly as the bending load increases. On the other hand, we noted that the voltage output of $\left(\sigma_{4}+\sigma_{4}{ }^{\prime}\right)$ is $0 \mathrm{~V}$, uninfluenced by the bending load, and that the compressive component is canceled. The results of Figs. 3 and 4 confirmed that this measurement method is able to measure only the bending load and compressive load components.

As shown in Fig. $5(\mathbf{a})$, in order to evaluate contact point $l$, we next applied a $98 \mathrm{~N}$ point load to the inner piece surface, moved the load position in the $\mathrm{X}$ and $\mathrm{Y}$ directions, and investigated the relationship between the actual contact position $\mathrm{L}$, and $l$ as calculated using Equation (12). As Fig. 5(b) shows, even if the distance $d$ along the $\mathrm{Y}$ direction shifts \pm $5 \mathrm{~mm}$ from the inner piece center, it remains very close, within $\pm 0.2 \mathrm{~mm}$, to the value of $l$ obtained according to the load position. This confirmed that the method is capable of accurately measuring the load's mechanical action origin.

\subsection{Powder and Experimental Method 2.3.1 Experiment with Glass Beads}

We installed the inner piece of Fig. 2 in the mechanofusion apparatus shown in Fig. 1 and

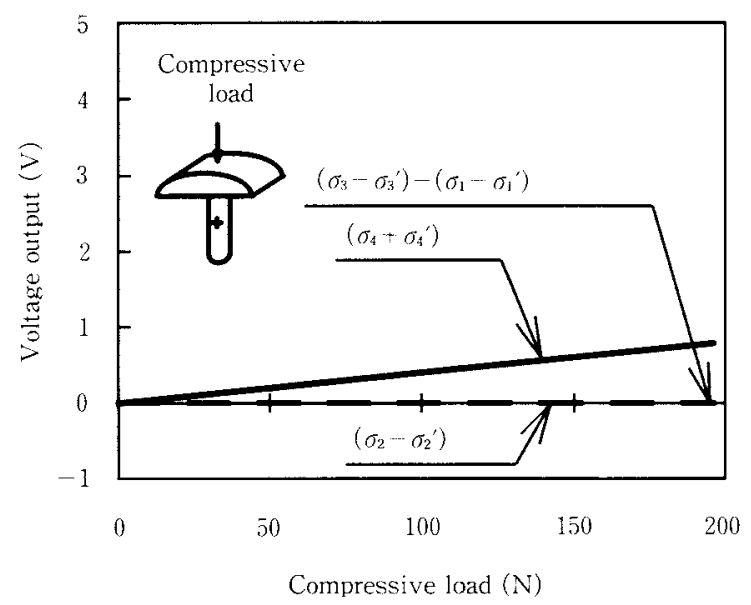

Fig. 3 Linear relationship between compressive load to the arm of inner piece and voltage output from strain gauges.

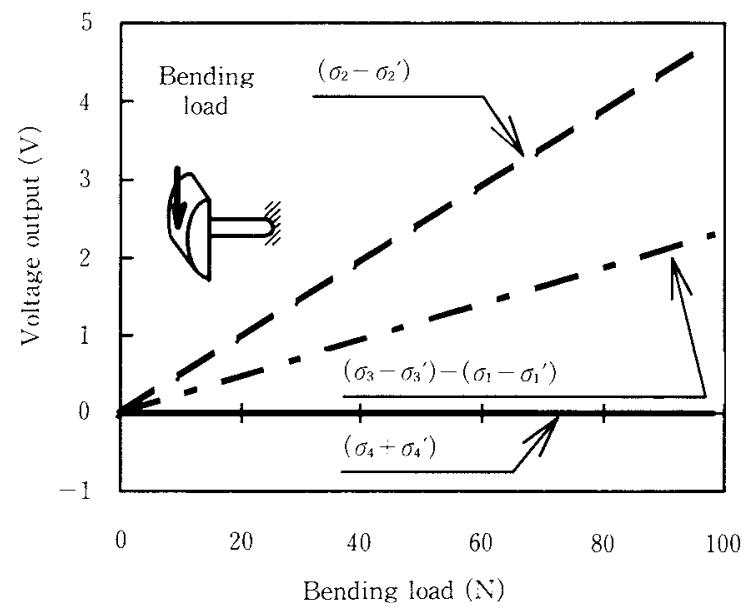

Fig. 4 Linear relationship between bending load to the arm of inner piece and voltage output from strain gauges. 
Mmechanically processed glass beads whose average diameter was $100 \mu \mathrm{m}$. The processing atmosphere was air. We loaded the apparatus with $100 \sim 200 \mathrm{~g}$ ( $70 \sim 140 \mathrm{cc}$ ) of glass beads, and set the gap between the drum and inner piece at a constant $2 \mathrm{~mm}$. Drum rotational speed was $10 \sim 23.3 \mathrm{~s}^{-1}$, and was changed in stages of $3.3 \mathrm{~s}^{-1}$ between these two speeds. We had determined in a preliminary experiment that this rotational speed range was below the limit at which the glass beads would be pulverized.

Simultaneously measured items were compressive force, frictional force, contact position between glass beads and inner piece, temperature of strain gauges, drum rotational speed, and power consumption of the motor that rotates the drum. The values were obtained by averaging the values over periods of about 30 seconds after the drum stabilized at a new rotational speed. There were no major varia tions in measured values during these time periods.

\subsubsection{Experimental Method Using Mixed Powder}

We measured forces during mechanical composite processing in the same manner as in section 2.3. 1. Powders used in the experiment were the same as in a previous report ${ }^{5}$ : spherical $\mathrm{Cu}$ core particles Inner piece
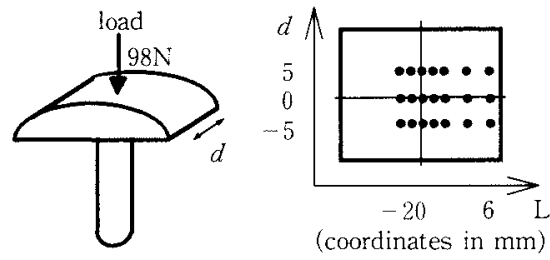

d,L : Distance from center line

(a)

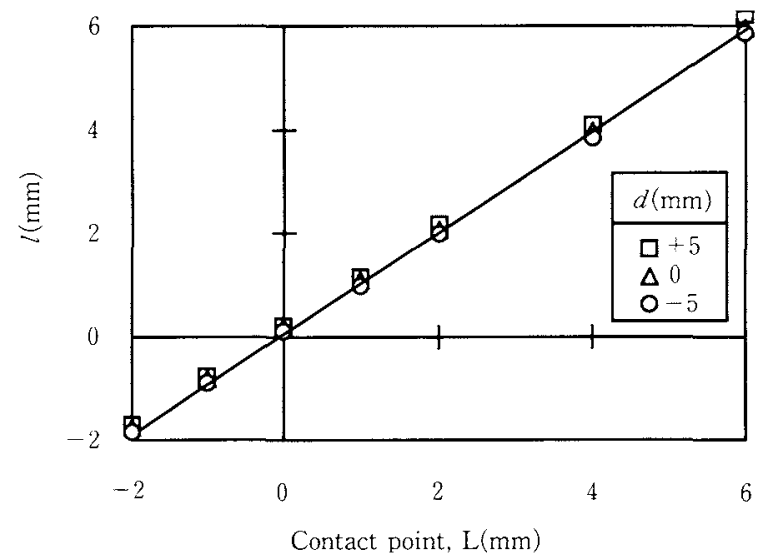

(b)

Fig. 5 The points as shown in the top view, loaded $98 \mathrm{~N}$ on the inner piece head (a), and coincidence of the distances to the points under actual loads and calculated from strain. with average diameters of $30 \mu \mathrm{m}$, and $0.5 \mu \mathrm{m}$ $\alpha \cdot \mathrm{Al}_{2} \mathrm{O}_{3}$ adhering particles. We loaded the drum with $200 \sim 400 \mathrm{~g}(38 \sim 76 \mathrm{cc})$ of a mixed powder comprising 2 mass $\% \mathrm{Al}_{2} \mathrm{O}_{3}-98$ mass $\% \mathrm{Cu}$ (below, $2 \mathrm{Al}_{2} \mathrm{O}_{3}-98 \mathrm{Cu}$ ), and subjected the mixture to composite processing at rotational speeds of $6.7 \sim 23.3 \mathrm{~s}^{-1}$ after evacuating the drum to prescribed pressures of $5.0 \times 10^{-2} \mathrm{~Pa}$ (high vacuum), $5.0 \times 10^{2} \mathrm{~Pa}$ (low vacuum), and $1.0 \times 10^{5} \mathrm{~Pa}$ (atmospheric pressure). Items measured during processing and the method of measurements were the same as with glass beads in section 2.3.1.

\section{Results and Discussion}

\subsection{Measurements with Glass Beads \\ 3.1.1 Effects of Rotational Speed}

Fig. 6 shows the changes in frictional force and compressive force corresponding to drum rotational speed and the amount of glass beads loaded. Frictional force and compressive force increase linearly as rotational speed increases. At the same rotational speed, the more glass beads loaded, the higher the frictional and compressive forces. How. ever, frictional and compressive forces increase to about the same extent with rotational speed. We found that compressive force is about eight times greater than frictional force.

If one assumes friction between the inner piece

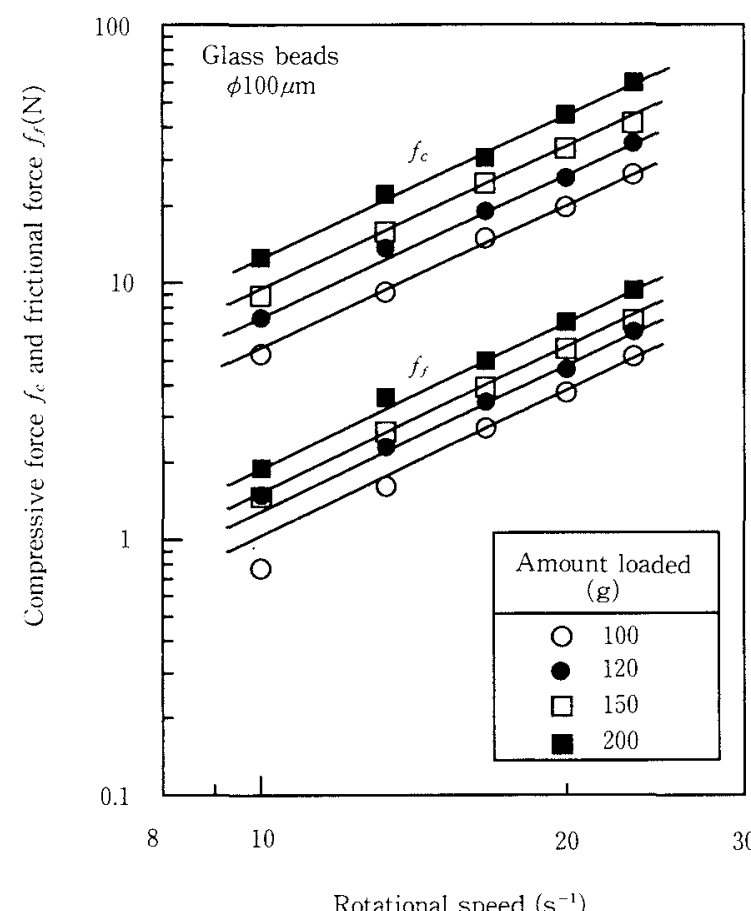

Fig. 6 Variation in frictional and compressive forces accord ing to rotational speed of drum, as a parameter of the amount of glass beads loaded. 
Pandoylass beads in accordance with Coulomb's law ${ }^{10}$, the relation between compressive force and frictional force is shown by the following equation.

$$
f_{f}=\mu \cdot f_{c}
$$

Here $\mu$ is the coefficient of friction, whose determination has nothing to do with rotational speed. We used Equation (13) to find $\mu$, and plotted it against rotational speed. Results appear in Fig. 7, which shows that the coefficient of friction is nearly a constant value that is not dependent on rotational speed. These results support the soundness of our measured values for frictional force.

Fig. 8 shows the change in the mechanical action origin $l$, where the inner piece and the powder make contact, as a function of rotational speed. $l$ falls as rotational speed increases, and becomes nearly constant beginning at a certain speed. The greater the amount of glass beads loaded, the bigger $l$ becomes.

This shows that the higher the rotational speed, and the lower the amount of beads loaded, the more

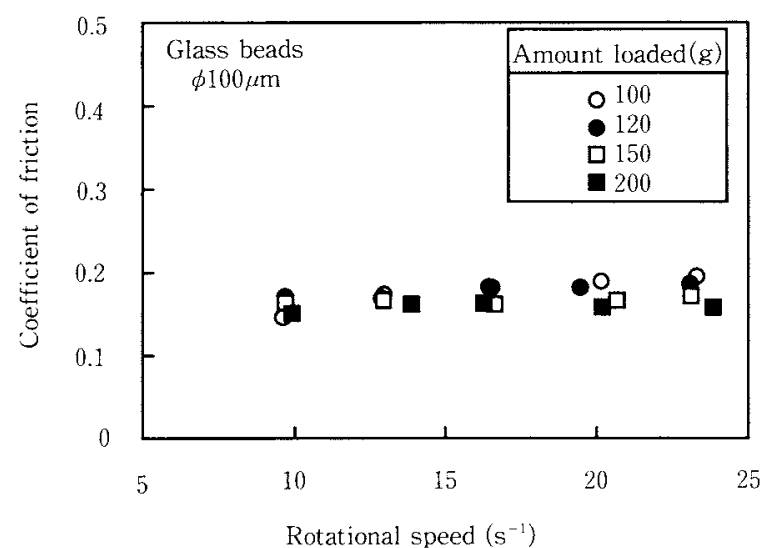

Fig. 7 Variation in the coefficient of friction acoording to rotational speed of drum, as a parameter of the amount of glass beads loaded.

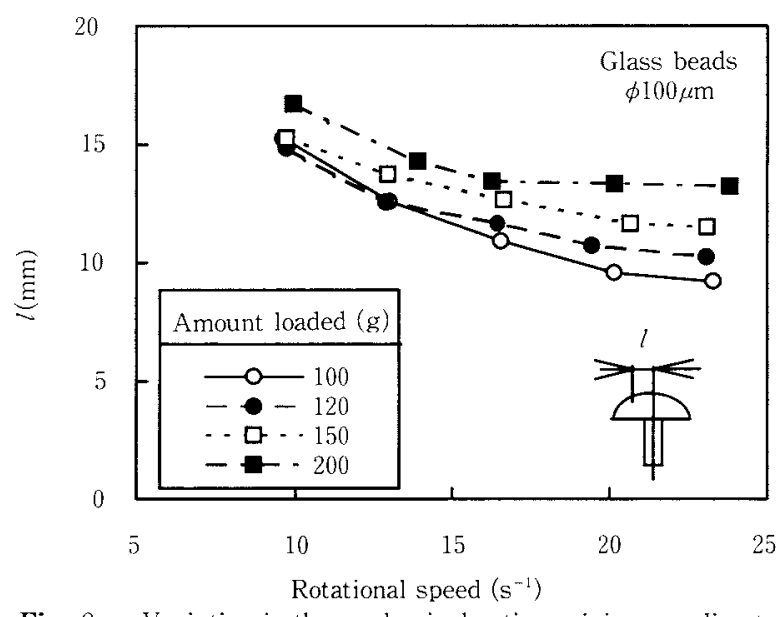

Fig. 8 Variation in the mechanical action origin according to rotational speed of drum, as a parameter of the amount of glass beads loaded. the contact location approaches the inner piece's center. One conceivable reason that this position approaches the center with a low bead load is a simple geometrical factor: because the amount of beads carried between the inner piece and drum is small, the thickness of beads pressed against the drum's interior wall is small. In terms of rotational speed effect, however, the reason is probably as follows. Because the centrifugal force acting on the beads is weak at low rotational speeds, they collect on the lower part of the drum wall. In effect this is conceivably the same situation as when the glass bead load is large, and a thick layer of beads is pressed against the drum wall, because of which the contact location moves away from the inner piece's center.

On the other hand, at high rotational speeds a strong centrifugal force acts on the glass beads, producing a stronger force pressing the beads against the drum wall, and pushing them up even against the top portion of the wall, which forms the beads into a thin layer. We surmise that as a result, the contact location with the glass beads approached the inner piece's center.

\subsubsection{Effects of Amount Loaded}

Fig. 9 shows the change in compressive force as a function of the amount of glass beads loaded. There is a linear proportional relationship between the amount loaded and compressive force, in which compressive force increases with the amount loaded. The extent to which compressive force increases as the amount loaded increases will differ according to drum rotational speed : the higher the rotational speed, the larger will be its rate of

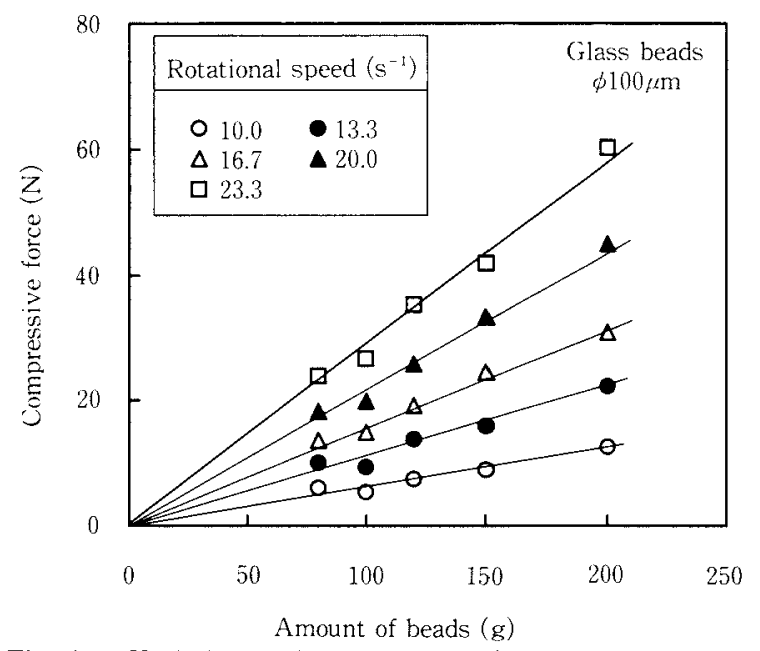

Fig. 9 Variation in the compressive force generated by the amount of glass beads, as a parameter of the rotational speed of the drum. 
Pricrasse. This shows that compressive force per unit volume of powder for each rotational speed is constant. In other words, the total compressive force acting on the inner piece increases when the load of beads increases, but there is no change, per unit of powder loaded, in the mechanical energy needed for compositing.

However, this applies to the loaded amount of powder as a whole, because the energy expended on each individual particle differs according to factors such as surface area of contact and the movement of particles. Research results ${ }^{11}$ show that in compositing experiments with $\mathrm{Cu}-\mathrm{Al}_{2} \mathrm{O}_{3}$ mixed powder, composite particles assume different forms, even at the same rotational speed, depending on the amount of powder loaded, and that as the amount loaded increases, particles increasingly exhibit the kind of composite behavior observed at high rotational speeds. When in this way conceiving the mechanical energy expended on compositing in terms of total compressive force, there is a contradiction between this and actual compositing phenomena. If one is going to rigorously examine compositing behavior in relation to mechanical behavior, it will be necessary to elucidate the stress acting on a powder bed. Quantifying that stress will necessitate a detailed analysis in terms of the contact area size and stress distribution of the inner piece and powder bed, as well as the dynamic behavior of the powder itself.

\subsubsection{Power Consumption}

To examine the soundness of this mechanical measurement method, we compared the measured value for net electric power consumed during processing with the motive power calculated from the frictional force and bending stress acting on the inner piece (below called the calculated value of power consumed), which were determined in the previous sections.

Here net power consumed means the electric power value when rotating the mass of the glass beads (the value of power used only for rotation as measured when imposing the mass of the glass beads on the drum) subtracted from the electric power value when processing the glass beads. Usually, in order to check agreement with calculated values, it would also be necessary to subtract, from this net power consumption, the power required by the scraper to dislodge powder from the drum wall. In this case, however, we ignored this amount of power as very small because the spherical glass

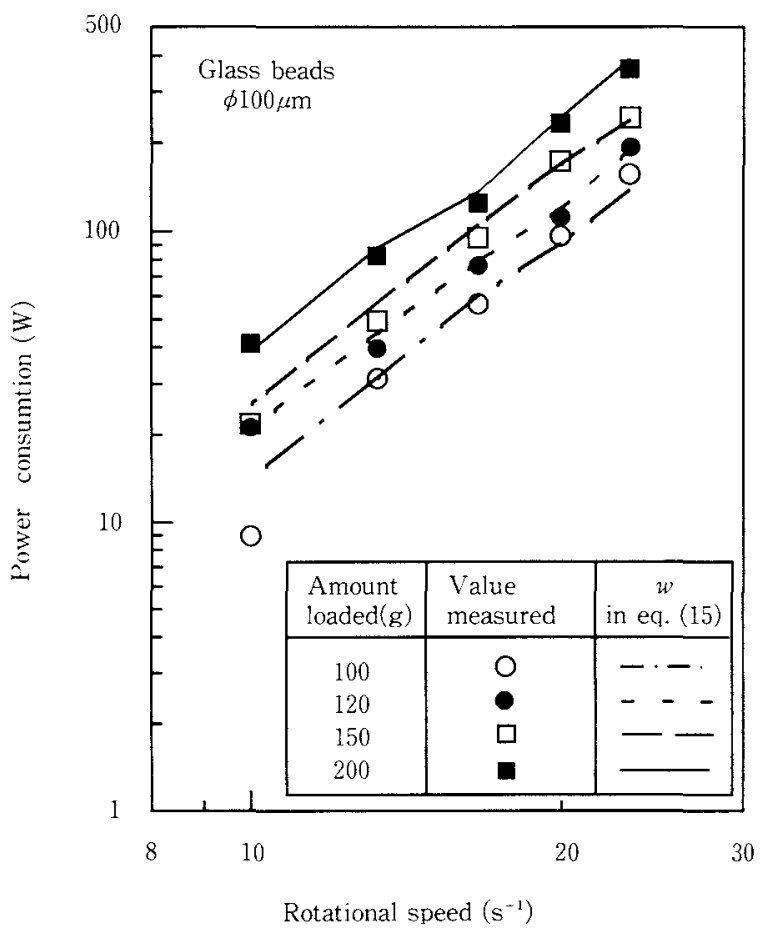

Fig. 10 Variation in power consumption with drum rotational speed as a parameter of amount of glass beads. The symbols and lines correspond to the measured values and the lines calculated by Eq. (15), respectively.

beads have low compaction and high fluidity.

Equations (14) and (15) convert to motive power $P(\mathrm{~kg} \cdot \mathrm{m} / \mathrm{s})$ and electric power $w(\mathrm{~W})$. Here $T$ is the torque determined by the vertical component of force along the inner piece arm $\left(F_{f}\right)$ that is imposed on the inner piece head and the drum radius, while $\mathrm{n}$ is drum rotational speed.

$$
\begin{gathered}
P=T \cdot 2 \pi \cdot n \\
w=P \cdot 9.8
\end{gathered}
$$

Fig. 10 shows net electric power and motive power calculated with Equation (15), plotted as a function of rotational speed. Lines are calculated values, and points are measured net power used. Calculated and measured values are very close in all four bead loading amounts. This corroborates the fact that the method used through the previous section to measure forces can accurately detect the forces generated during processing, which suggests that we were right in assuming it is all right to ignore the power consumed by the scraper, as mentioned above.

\subsection{Measurements with $\mathrm{Al}_{2} \mathrm{O}_{3}$ - $\mathrm{Cu}$ Mixed Powder}

\subsubsection{Changes in Strength due to Residual Pres- sure}

We loaded $2 \mathrm{Al}_{2} \mathrm{O}_{3}-98 \mathrm{Cu}$ mixed powder into the drum and exhausted it to the prescribed residual 
pnessure, then performed composite processing at drum rotational speeds of 6.7 to $23.3 \mathrm{~s}^{-1}$. Fig. 11 shows the frictional force and compressive force measured while processing. Powder loaded was 400 $\mathrm{g}$, and measurement results were taken over 300 seconds from the start of processing. Frictional force and compressive force increase as rotational speed increases. At the same rotational speed, frictional force and compressive force tended to increase as pressure fell, and the higher the rotational speed, the more marked the increase in frictional and compressive forces in relation to pressure.

Fig. 12 shows the changes in mechanical action origin $l$ in conjunction with pressure. It contrasts the results obtained at 60 and 300 seconds after the start of compositing with loads of 200 and $400 \mathrm{~g}$. Rotational speed was $11.7 \mathrm{~s}^{-1}$. The value of mechanical action origin $l$ becomes smaller as pressure drops. Also, the value of $l$ was larger for the $400 \mathrm{~g}$ powder load than for $200 \mathrm{~g}$. This value is smaller at 300 seconds after the start of processing than at 60 seconds, meaning that the contact location with the powder was approaching the inner piece center.

The reasons are as follows. When much powder is loaded, a large volume of powder is brought between the inner piece and the drum. Because a thick powder bed is formed by centrifugal force pressing powder against the drum wall, the value of mechanical action origin $l$ becomes large, thus showing that the contact position moves away from the inner piece's center.

Next we shall examine the effects of residual pressure on $l$. The particles that scatter when dislodged from the drum wall by the scraper either directly reach the wall or reach the bottom of the drum by sedimentation or the like, and move to the drum wall by centrifugal force. Particles are subject to fluid resistance, a force acting opposite to their direction of movement. This fluid resistance is proportional to the product of projection area to the direction of particle movement, and kinetic energy $\rho \cdot v^{2} / 2{ }^{12}$ Here $v$ is particle and fluid relative speed, and $\rho$ is fluid density. Because fluid density $\rho$ is proportional to residual pressure, fluid resistance is higher at atmospheric pressure than under a high vacuum. This shows that the the lower the residual pressure, the faster particles move, and the less time it takes for them to reach the drum wall. Thus the amount of powder stuck to the drum wall per unit time becomes larger, but as the powder's force for climbing vertically through the drum is also large, in effect it is the same as if the powder bed on the interior wall were thin. This we can see as being equivalent to the phenomenon in Fig. 11, wherein frictional and compressive forces increase under low residual pressure. Conceivable causes for the decrease in $l$ under low residual pressure include decreased volume of powder between the inner piece and the drum owing to increased tapping density caused by vacuum, and higher fluidity due to

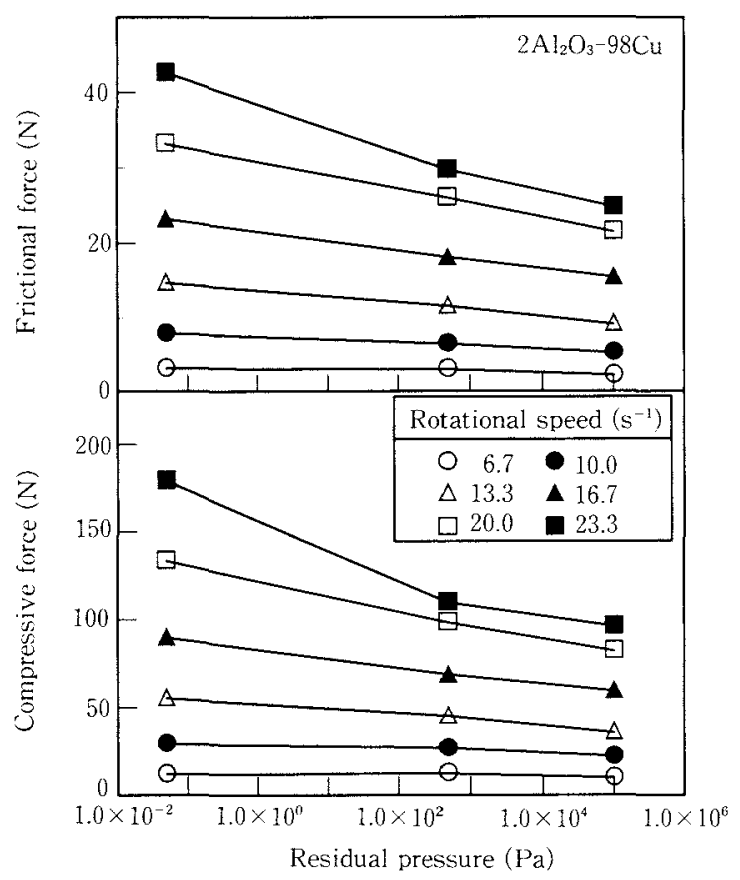

Fig. 11 Variation in the compressive and frictional forces for various residual pressures at the processing time of 300 s, as a parameter of the rotational speed of drum, for $2 \mathrm{Al}_{2} \mathrm{O}_{3}$ 98 $\mathrm{Cu}$ mixed powder.

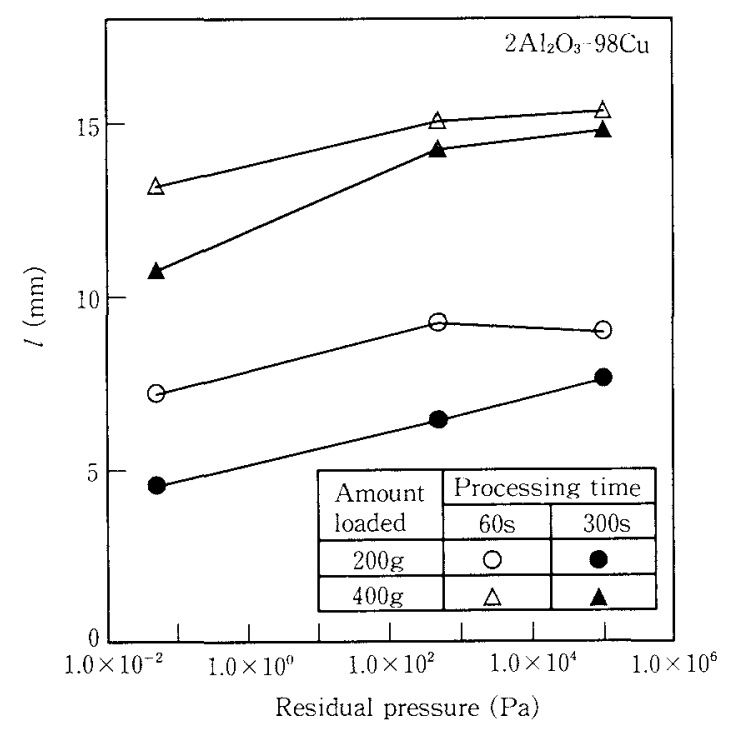

Fig. 12 Change in the mechanical action origin at various residual pressures, as a parameter of the amount of $2 \mathrm{Al}_{2} \mathrm{O}_{3}-98 \mathrm{Cu}$ mixed powder and the processing time. 
vacum drying, but this is still not clear. $l$ is smaller at 300 seconds than at 60 seconds, the reason being perhaps that specific surface area decreases as compositing proceeds, which results in a decrease in the thickness of the mixed particle bed on the drum wall. The authors surmise that the change in $l$ was large because compositing proceeds faster under a high vacuum than at atmospheric pressure.

Fig. 13 shows the power consumption 300 seconds

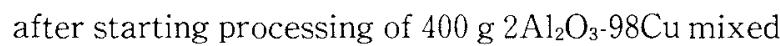
powder in relation to residual pressure and drum

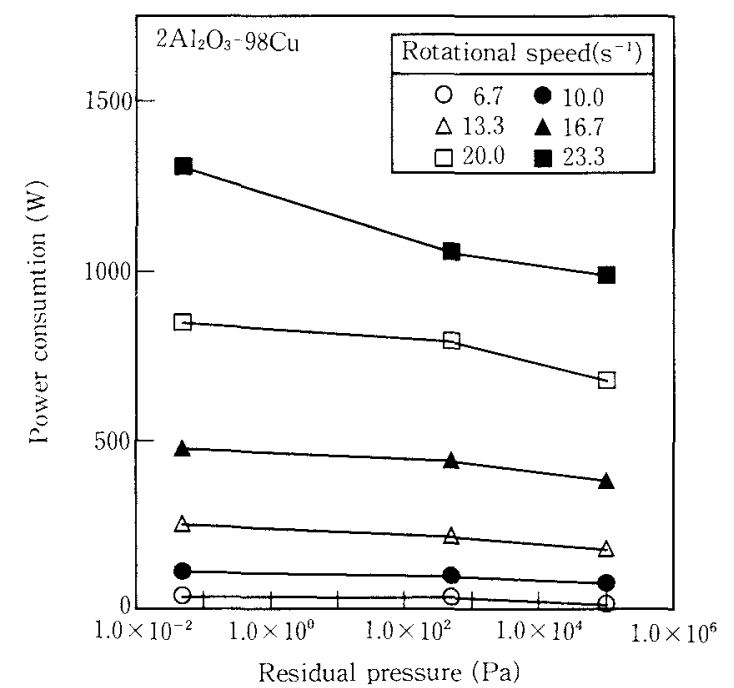

Fig. 13 Variation in power consumption at various residual pressures, as a parameter of the rotational speed of drum with constant amount $(400 \mathrm{~g})$ of $2 \mathrm{Al}_{2} \mathrm{O}_{3}-98 \mathrm{Cu}$ mixed powder.

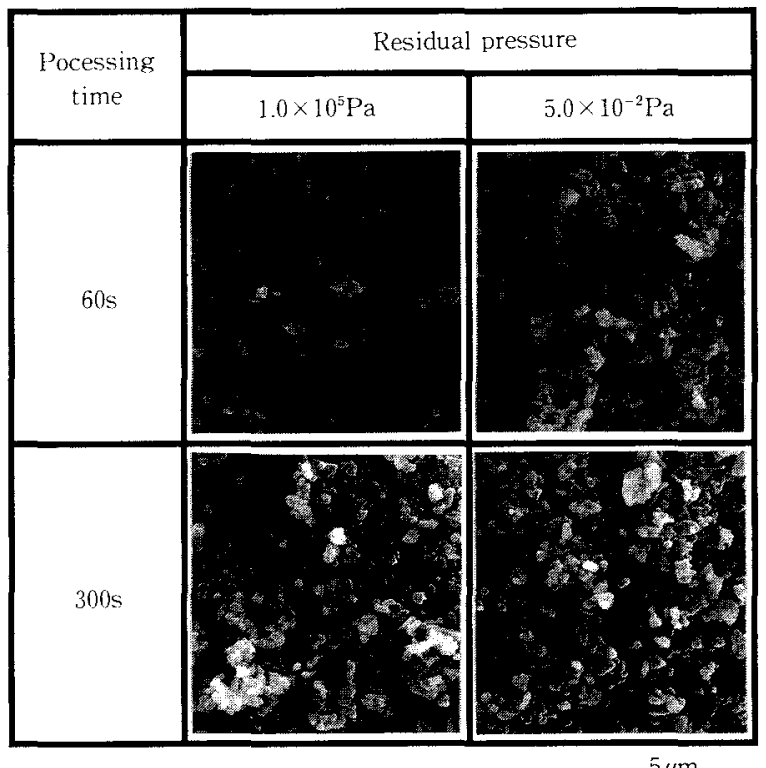

$5 \mu \mathrm{m}$

Fig. 14 Micrographs of the surface layers of the composite particles obtained from $2 \mathrm{Al}_{2} \mathrm{O}_{3}-98 \mathrm{Cu}$ mixed powder by changing the residual pressure and processing time rotational speed. Power consumption here is the value obtained by subtracting the consumption required to simply rotate mixed powder of $400 \mathrm{~g}$ mass. The higher rotational speed, the more power is consumed. Meanwhile, at the same rotational speed, power consumption rises as residual pressure declines, with the change in the rate of power consumption rise increasing as rotational speed increases.

After performing mechanical measurements on the $2 \mathrm{Al}_{2} \mathrm{O}_{3}-98 \mathrm{Cu}$ mixed powder, we made SEM observations of the composite particles' surfaces. These appear in Fig. 14. The minute surface parti. cles are the $\mathrm{Al}_{2} \mathrm{O}_{3}$ adhering particles. $\mathrm{Al}_{2} \mathrm{O}_{3}$ dispersion and adherence have already begun 60 seconds after the start of processing, but 300 seconds after the start of processing the adherence of $\mathrm{Al}_{2} \mathrm{O}_{3}$ particles has advanced even more than after 60 seconds. Also, at the same processing time, $\mathrm{Al}_{2} \mathrm{O}_{3}$ adheres better and appears to composite faster under a high vacuum than at atmospheric pressure. As in Fig. 11, a high vacuum is more effective than atmospheric pressure in generating frictional force and compres. sive force during composite processing, and makes compositing proceed better. With the added effect by which $\mathrm{Cu}$ oxidation is suppressed as previously reported $^{7}$, compositing proceeds rapidly under a vacuum.

\section{Conclusion}

We developed a method of measuring the compressive force and frictional force generated on the inner piece during mechanical processing, and used this method to conduct basic mechanical measuring tests during processing with glass beads. We also conducted mechanical measurements on $2 \mathrm{Al}_{2} \mathrm{O}_{3}$ $98 \mathrm{Cu}$ mixed powder when processing under a vac uum, and studied how these forces are associated with how compositing proceeds. These tests revealed the following items.

(1) From the stress determined by the strain gauges on the inner piece's arm, it was possible to measure the compressive force and frictional force arising between the powder being mechanically processed and the inner piece, as well as the distance to the mechanical action origin at which those forces arose.

(2) The higher the drum's rotational speed, the greater the compressive force and frictional force generated on the inner piece. Increasing the amount of powder loaded especially increases 
corhpressive force, but there is no change in compressive force per unit mass. As rotational speed increases, the mechanical action origin approaches the center of the inner piece.

(3) The electric power directly measured from the motor while processing powder closely matched the motive power value calculated from the mechanically measured value, which confirmed the soundness of these mechanical measurements.

(4) The compressive force and frictional force generated on the inner piece head differ according to residual pressure. As residual pressure drops, these forces become greater and the contact location between the inner piece head and the powder approaches the center of the inner piece head. The lower the residual pressure, the higher the power consumption during composite processing.

(5) After only 60 seconds from the beginning of composite processing the mixing/dispersion and compositing of adhering particles begins, and compositing proceeds faster under a vacuum than at atmospheric pressure.

Finally, we would like to express our appreciation to Chief Researcher Masaru Otani at the Hokkaido Industrial Research Institue for his cooperation in our experiments.

\section{Nomenclature}

$f_{f}$ : frictional force

$f_{c}$ : compressive force

$F_{f}$ : vertical component of $f_{f}$ and $f_{c}$

$F_{c}$ : horizontal component of $f_{f}$ and $f_{c}$

$\sigma_{n}, \sigma_{n}{ }^{\prime}(n=1 \sim 4):$ stress
$A:$ cross-sectinal area of shaft $\quad\left(\mathrm{mm}^{2}\right)$

$Z:$ modulus of section $\left(\mathrm{mm}^{3}\right)$

$R:$ radius of curvature of inner piece $(\mathrm{mm})$

$\mu:$ coefficient of friction

$P:$ motive power

$n:$ rotational speed of drum

$(\mathrm{kg} \cdot \mathrm{m} / \mathrm{s})$

$\left(\mathrm{s}^{-1}\right)$

$T:$ torque

$w$ : electric power

$(\mathrm{kg} \cdot \mathrm{m})$

\section{References}

1) Yokoyama, T., Urayama, K. and Naito, M.: Funtai to Kogyo, Japan, 19, 47 (1987)

2) Tanno, K. and Yokoyama, T. :J. Soc. Powder Technol., Japan, 27, 153 (1990)

3) Naito, M., Kondo, A. and Yokoyama, T.: ISIJ Intenational, 33, 915 (1993)

4) Kaga, H., Shimono, I., Taya, Y., Takamura, T., Sugawara, T. and Katayama, H.: J. Japan Soc. Powder and Powder Metallurgy, Japan, 37, 995 (1990)

5) Kaga, H., Tara, Y., Shimono, I. and Katayama, H. : ibid., 39, 546 (1992)

6) Kaga, H., Taya, Y., Katayama, H. and Hamaguchi, Y.: ibid., 39, 553 (1992)

7) Kaga, H., Taya, Y., Hamaguchi, Y., Katayama, H. and Mukaida, K.: Kona, 12, 145 (1994)

8) Naito, M. : Funtai to Kogyo, Japan, 21, 77 (1989)

9) Kanno, A.: "Ohryoku Hizumi Kaiseki”, p.41, Asakura Syoten (1986)

10) Moriguchi, S.: "Shotou Rikigaku”, p.171, Baifukan (1959)

11) Kaga, H., Taya, Y. : Report of the Hokkaido Industrial Technology Center, 2, 27 (1992)

12) Takahashi, K. : "Kiso Aerosol Kougaku", p.10, Youken do (1982) 


\section{Author's short biography}
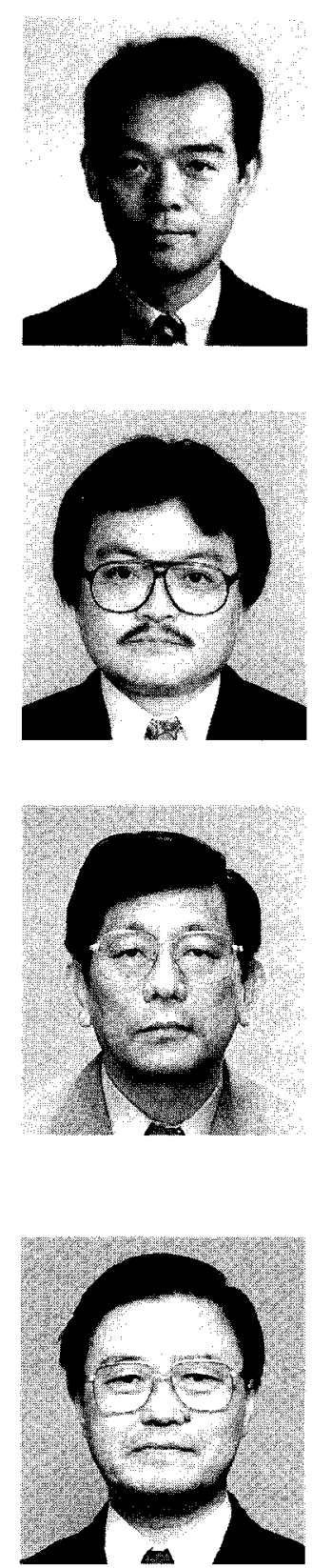

\section{Hiroyuki Yoshino}

Hiroyuki Yoshino studied mechanical engineering at the Akita University of Japan. He joined a motor company to design the motor car for 6 years.

Since 1987, he has been working at the Hokkaido Industrial Technology Center. He is a chief at the Department of Mechanical Engineering and has been mainly researching and developing new technologies of marine machinery. He has also been involved in the project of quantitative measurement of forces acting on a powder bed during a specific mechanical process since 1991.

\section{Yoshihiro Taya}

Yoshihiro Taya graduated in the Department of Chemical Engineering from Doshisha University. He did research and development at the air conditioning machinery company for 4 years. He joined the Department of Research and Development at Hokkaido Industrial Technology Center from 1986, and has been studying on the vacuum machineries.

\section{Dr. Hisashi Kaga}

Hissashi Kaga graduated in Metallurgical Engineering from Muroran Institute of Technology, and subsequently obtained and MEn from Tohoku University. After 13 years in the research institute of the steel company, he joined the department of research and development at Hokkaido Industrial Technology Center since 1986. He obtained his doctorate (1993) concerning physical preparation of composite particles. His major research interests are the preparation of the composite particles by physical processing and the preparation of high performance materials from the composite particles.

\section{Prof. Dr. Ken-ichi Mukaida}

Ken-ichi Mukaida graduated in Chemical Engineering from Muroran Institute of Technology (MIT) in 1961 and the graduate school of Engineering of Hokkaido University in 1963. He became an assistant professor of the Department of Chemical Engineering at MIT starting the research of the preparation, characterization, and modification of solid acid catalysts. He shifted to the department of Materials Science and Engineering in 1990, and thereafter he studies surface modification, metal-oxide composite physical-preparation and solid superacid catalysts. 